\title{
Facilitating Social Play for Children with PDDs: Effects of Paired Robotic Devices
}

\author{
Soichiro Matsuda ${ }^{1,2 *}$, Eleuda Nunez ${ }^{1}$, Masakazu Hirokawa ${ }^{1}$, Junichi Yamamoto ${ }^{3}$ and \\ Kenji Suzuki ${ }^{1}$
}

${ }^{1}$ Artificial Intelligence Laboratory, Faculty of Engineering, Information and Systems, University of Tsukuba, Tsukuba, Japan,

${ }^{2}$ Japan Society for the Promotion of Science, Tokyo, Japan, ${ }^{3}$ Department of Psychology, Keio University, Tokyo, Japan

OPEN ACCESS

Edited by:

Bilge Mutlu,

University of Wisconsin-Madison,

United States

Reviewed by:

Alda Troncone,

Università degli Studi della Campania

"Luigi Vanvitelli" Caserta, Italy

Charlotte Magnusson,

Lund University, Sweden

*Correspondence:

Soichiro Matsuda

matsuda@ai.iit.tsukuba.ac.jp

Specialty section:

This article was submitted to Human-Media Interaction, a section of the journal

Frontiers in Psychology

Received: 31 December 2016 Accepted: 06 June 2017

Published: 28 June 2017

Citation:

Matsuda S, Nunez E, Hirokawa M Yamamoto J and Suzuki K (2017) Facilitating Social Play for Children with PDDs: Effects of Paired Robotic

Devices. Front. Psychol. 8:1029. doi: 10.3389/fpsyg.2017.01029
Interacting with toys and other people is fundamental for developing social communication skills. However, children with autism spectrum disorder (ASD) are characterized by having a significant impairment in social interaction, which often leads to deficits in play skills. For this reason, methods of teaching play skills to young children with ASD have been well documented. Although previous studies have examined a variety of instructional strategies for teaching skills, few studies have evaluated the potential of using robotic devices. The purpose of the present study is to examine whether automatic feedback provided by colored lights and vibration via paired robotic devices, COLOLO, facilitates social play behaviors in children with ASD. We also explore how social play relates to social interaction. COLOLO is a system of paired spherical devices covered with soft fabric. All participants in this study were recruited as volunteers through the Department of Psychology at Keio University. The pilot study included three participants diagnosed with Pervasive Developmental Disorders (PDDs; 5- to 6-yearold boys), and compared experimental conditions with and without automatic feedback from the devices (colored lights and vibration). The results indicated that the participants in the condition that included feedback from the devices exhibited increased rates of ball contact and looking at the therapist's ball, but did not exhibit increased rates of eye contact or positive affect. In the experimental study, a systematic replication of the pilot study was performed with three other participants diagnosed with PDDs (3- to 6year-old boys), using an A-B-A-B design. Again, the results demonstrated that, in the condition with colored lights and vibration, the children increased ball contact as well as looking at the therapist's ball. However, the results did not show the effect of automatic feedback consistently for three children. These findings are discussed in terms of the potential of paired robotic devices as a method to facilitate social play for children with ASD.

Keywords: autism spectrum disorder (ASD), social play, paired robotic devices, children, robot-mediated therapy, single subject design

\section{INTRODUCTION}

Difficulties with play skills have been well documented in children with Autism Spectrum Disorder (ASD; Wuff, 1985; Baron-Cohen, 1987; Lewis and Boucher, 1988; Jarrold et al., 1993; Charman et al., 2000; Williams et al., 2001). These difficulties are seen in sensory motor play, manipulative play, physical play, pretend play, and social play (Boucher, 1999). Consistent with this view, many studies have focused on teaching play skills to children with ASD (Jung and Sainato, 2013). 
Previous intervention studies have used video and live modeling (Jahr et al., 2000; MacDonald et al., 2005), pivotal response training (Stahmer, 1995; Thorp et al., 1995), activity schedules (Morrison et al., 2002; Machalicek et al., 2009), or social stories (Barry and Burlew, 2004). Researchers have also combined these strategies with contingent reinforcement (Jung and Sainato, 2013). These studies have found training increases engagement in appropriate play behavior and cooperative play in children with ASD. On the other hand, few studies have examined the effectiveness of robotic device use in teaching play skills to children with ASD, although robotic devices can automatically and immediately reinforce appropriate play behavior.

Robotic devices have been used to increase socialcommunication behaviors, such as joint attention (Warren et al., 2015; Simut et al., 2016) and imitation (Duquette et al., 2008), in children with ASD. These studies have focused on the use of both humanoid robots and non-humanoid toy-like robots, such as KASPER (Robins et al., 2009), Keapon (Costescu et al., 2015), NAO (Huskens et al., 2015; Warren et al., 2015), Probo (Simut et al., 2016), Robota (Billard et al., 2007), or Tito (Duquette et al., 2008). However, these robots mainly provide feedback as a result of the behavior of a child. Given that the facilitation of social play involves two people using toys, it may be necessary to consider directly providing feedback as a result of the behavior of both a child and the other individual.

Paired robotic devices might encourage cooperative behaviors, such as turn taking (Nunez et al., 2016). In this approach, remotely connected paired devices provided feedback separately as a result of child's own behavior as well as the other individual's behavior. Huskens et al. (2013) suggested robotic devices should be deployed as mediators to promote social interaction between a child with ASD and another individual. However, to our knowledge, no studies have examined how automatic feedback via paired robotic devices affects social play behaviors. In addition, as Diehl et al. (2012) have pointed out, most studies using robots for children with ASD have not used an experimental design, such as an experimental group design or single subject experimental design.

When considering play behaviors, we need to recognize two types. First are those related to social play, such as ball contact and looking at a therapist's ball (Bass and Mulick, 2007). Second are those related to social interaction, such as eye contact and positive affect. The purpose of the current study is to examine whether automatic feedback via paired robotic devices facilitates social play behaviors in children with ASD, and to explore how social play relates to social interaction.

If the paired robotic devices can immediately provide automatic feedback contingent on child's social play behaviors, it is possible that automatic feedback increases the social play behaviors in children with ASD. Therefore we hypothesized the following relationship between behavior contribution and feedback:

Hypothesis 1: The child's ball contact and looking at the therapist's ball will increase with automatic feedback in the form of vibration and light.
It is possible that social play will also facilitate social interaction, and then we could expect that:

Hypothesis 2a: The automatic feedback by vibration and light will increase behaviors associated with social interaction, such as eye contact and positive affect.

Alternatively, it is also possible that social play directs the child's attention away from the therapist toward the activity, and thus we could expect that:

Hypothesis 2b: Automatic feedback in the form of vibration and light will decrease behaviors associated with social interaction, such as eye contact and positive affect.

To directly test these hypotheses a single $A B$ design was used in a pilot study to make inferences about the effects of feedback made by colored lights and vibration via paired robotic devices on social play behaviors in three boys with PDDs. In this experiment, we used a rapidly changing reversal design with the same experimental condition as the pilot study. By using this experimental design, we further evaluated whether and what types of social play behaviors are facilitated by the feedback provided by remotely connected paired devices in children with PDDs.

\section{GENERAL METHOD}

\section{Paired Robotic Devices: COLOLO}

In the experiments, we used a system composed of paired devices, COLOLO. The devices have embedded sensors to detect when they are being manipulated, sending a message to the paired devices. This message is represented by visual cues made by colored lights and movements. Each device is made of a plastic spherical case covered by soft material. Inside there is a plate attached to the rotational axis of a motor by a microcontroller. A weight is attached to the motor and allows the sphere to wiggle by unbalancing the device. On the plate, there is a circuit board where a microcontroller, wireless communication module, tilt sensor, battery, and full color LEDs are installed. Each device is connected to a server via TCP/IP protocol. The server is a stationary computer that identifies the client device by a predefined ID. The roles of the server are to mediate communication among clients, pair/group clients, and log clients' communication history. The microcontroller changes the color of the LEDs and sends a message to the server when the tilt sensor detects the user's manipulation. Then, when the paired devices receive the message, the sphere starts wiggling and the color of the LEDs change according to the information in the message. In this way, users can perceive others' actions by visualizing color changes and wiggling motions. More details on the device can be found in our previous work (Nunez et al., 2016).

\section{Experimental Condition}

Both conditions (with and without automatic feedback) were implemented on the carpeted floor of a testing room at a 
university. In order to improve the visibility of light under the feedback condition, direct illumination was turned off and indirect lighting set at the two corners of the room (Figure 1). All sessions were videotaped.

There were two experimental conditions. The first condition was the with automatic feedback condition (Phase A). The sensors embedded in the devices detected contact (e.g., handling, bouncing, or tossing) and displayed feedback using colored lights and vibration according to the interaction rule (Figure 2). Under this rule it is necessary to use two devices that send and receive messages triggered by the users actions (paired configuration). When the sender device is manipulated, the visual/tactile feedback is transferred to the receiver device. By doing this, the roles of the devices are switched. If a receiver device is manipulated, it will not respond to the actions until it receives the turn from the sender device. The second condition was the without automatic feedback condition (Phase B), in which the devices were turned off. Therefore, the child and the therapist used them as regular balls.

The study examined the differences in child social play behaviors within the two experimental conditions: with and without automatic feedback. In both conditions, the interaction took place in the following format: (1) the therapist introduced balls to the child; (2) the therapist modeled how to play with the balls (e.g., rolling, shaking, and catching them); (3) the child manipulated the balls; and (4) the child's ball manipulating behavior was verbally/physically praised by the therapist (e.g., "You're great!" and tickling). In addition, the therapist verbally/physically praised whenever the child made eye contact, exhibited positive affect, or approach to the therapist, throughout the session.

\section{Diagnosis Procedure}

This study was approved by the affiliate university's Institutional Review Board and was, therefore, completed in accordance with the ethical standards established in the 1964 Declaration of Helsinki. All participants had a diagnosis of autistic disorder, PDD-NOS, or ASD by an outside medical doctor. Diagnosis of Pervasive Developmental Disorders (PDDs) was further confirmed using the Pervasive Developmental Disorders Autism Society Japan Rating Scale (PARS; Kamio et al., 2006; Ito et al., 2012a). PARS, developed in Japan, is an interviewbased instrument for evaluating PDDs according to DSM-IV-TR (American Psychiatric and Association, 2000). The sub and total scores of PARS have correlations with the domain and total scores of the Autism Diagnostic Interview-Revised (ADI-R; Le Couteur et al., 1989; Lord et al., 1994). All participants with PDDs met the threshold for a diagnosis of PDDs on a total peak symptom scale score $(>9)$.

\section{Dependent Variables}

Four dependent variables (eye contact, positive affect, ball contact, and looking at the therapist's ball) were scored using occurrence/non-occurrence data in 15-s intervals. For each session, 20 intervals were recorded. Videotape scoring was completed by a scorer who was naïve to the purpose of the study. Eye contact: Eye contact was defined as the child's looking at the therapist's facial region. Positive affect: Positive affect was defined as visible and/or audible indications of happiness and enjoyment, including smiling and laughing. Ball contact: Ball contact was defined as the child's contact with the ball, including handling, bouncing, and tossing the ball. Looking at the therapist's ball: Looking at the therapist's ball was scored when the child was looking at the ball that the therapist held.

\section{Inter-observer Agreement}

Inter-observer agreements (i.e., agreements divided by agreements plus disagreements and multiplied by 100) were calculated for both the pilot study and the experimental study. The second observer was the first author, who independently scored 33\% (for pilot study) and 25\% (for experiment) of the sessions for four dependent variables. Agreement was calculated as the average percentage of agreement across sessions.

\section{Procedural Fidelity}

To assess the degree to which all sessions were executed according to procedure, reliability indices for fidelity of implementation (i.e., agreements divided by agreements plus disagreements and multiplied by total number of sessions) were collected for both the pilot study and the experimental study. A research assistant and the second author completed procedural fidelity checklist on three different variables for all sessions.

\section{PILOT STUDY}

\section{Participants}

All participants were recruited as volunteers through the Department of Psychology at Keio University. Participants were three boys with PDD, "Taro," "Sabu," and "Jiro," between the ages of 5 and 6 years. Names of participants have been changed to protect the participants' identities. Informed consent was obtained from the parents before the children were included in the study.

Table 1 displays the participants' characteristics. The participants' initial profiles (i.e., language, communication, motor, perceptual, and adaptive behavior skills) were assessed using standardized assessment tools: the Kyoto Scale for Psychological Development 2001 (KSPD; Ikuzawa et al., 2002), the Vineland Adaptive Behavior Scales, 2nd edition Japanese version (Vineland-II; Ito et al., 2012b), and the MacArthur Communicative Development Inventories, Japanese version (MCDIs; Ogura, 2007). The KSPD yields standard scores for physical-movement (P-M), language-sociability (L-S), and cognitive-adaptive (C-A) subscales and total developmental quotient (DQ). The KSPD was developed for use with typically developing infants and low-function children with ASD and other developmental disorders in Japan.

\section{Design and Procedure}

A single $A B$ design was used in the pilot study. By contrasting the with automatic feedback condition (Phase A) and the without automatic feedback condition (Phase B), we could make 


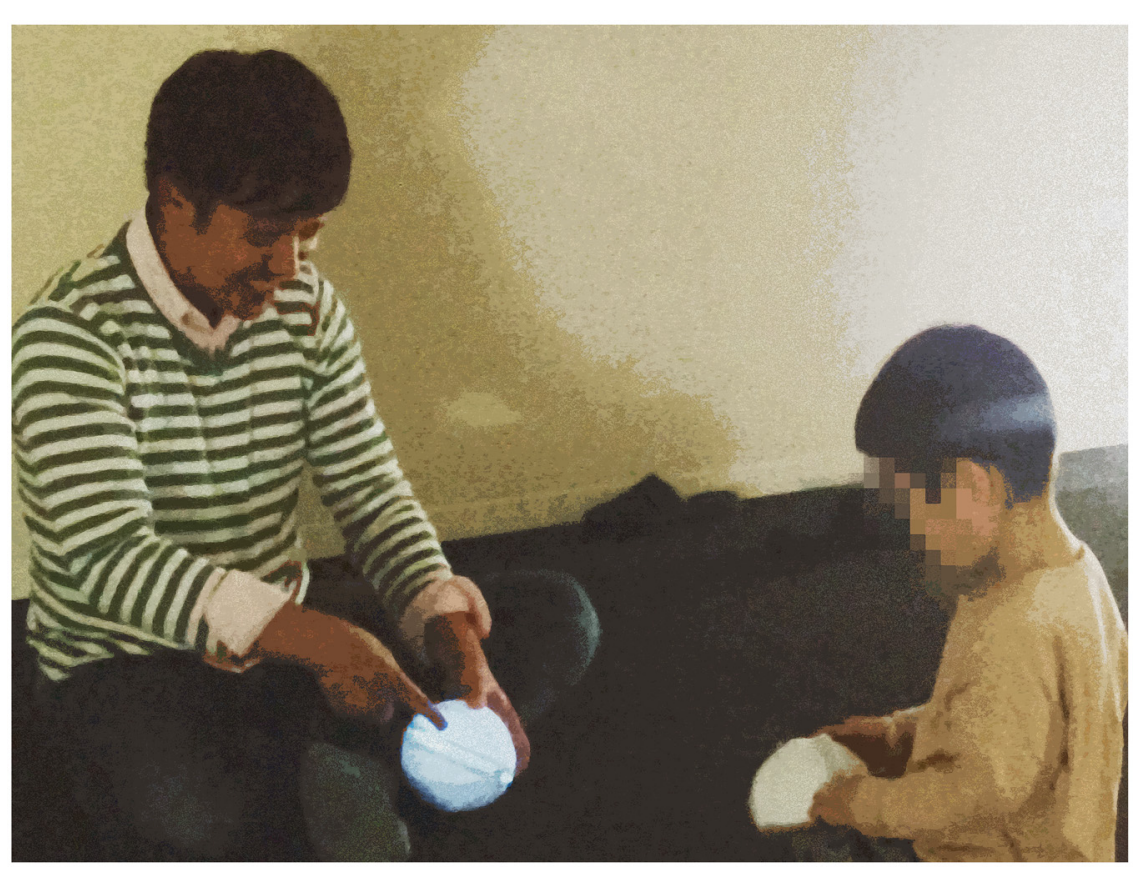

FIGURE 1 | Basic image of a session in the with automatic feedback condition. The therapist and the child both hold COLOLO.
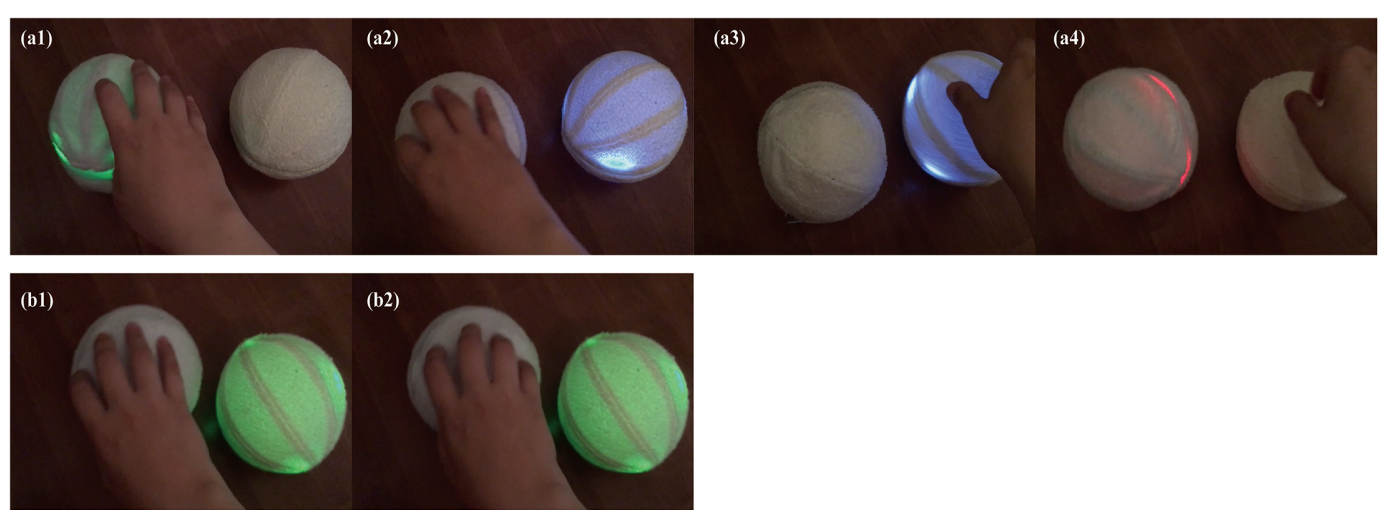

FIGURE 2 | Example of the transferring lights rule. (a1) When a user manipulates Device A, (a2) the paired device (Device B) will provide feedback of light and vibration. (a3) When the user manipulates Device B, (a4) the paired device (Device A) will provide feedback of light and vibration. (b1) When the user manipulates Device A during the feedback of Device B, (b2) Device A will not give any feedback, and the user will need to wait for a response.

inferences about differences of the dependent variables between the experimental conditions.

Each phase consisted of a 5-min session, and both phases were conducted in a same day for each participant. First, the with automatic feedback condition (Phase A) was presented, and then, after a short break, the without automatic feedback condition (Phase B) followed.

\section{Results}

For eye contact, the average observer agreement value was $97 \%$ (range 95-100\%); for positive affect, 97\% (range 95-100\%); for ball contact, $90 \%$ (range $85-95 \%$ ); and for looking at the therapist's ball, $82 \%$ (range $80-85 \%$ ). Fidelity of implementation for socially/physically reinforcing the child's eye contact, positive affect, and approach to the therapist averaged $67 \%$; fidelity of implementation for socially/physically reinforcing the child's ball contact averaged 100\%; and fidelity of implementation for modeling and prompting ball play averaged $100 \%$. Results are shown in Figure 3.

\section{Eye Contact}

The percentage of intervals with eye contact in the with automatic feedback condition was $0 \%$ for Taro, $0 \%$ for Jiro, and $15 \%$ for Sabu. On the other hand, in the without automatic feedback condition, these numbers increased to 10,15 , and $55 \%$, respectively. 
TABLE 1 | Participant profiles in the pilot study.

\begin{tabular}{llccc}
\hline Child & & Taro & Jiro & Sabu \\
\hline Chronological age & & $6 ; 9$ & $5 ; 6$ & $5 ; 6$ \\
PARS & Total peak symptom scale score & 51 & 28 & 24 \\
KSPD & Full DQ & 77 & 33 & 38 \\
& P-A DQ & 56 & 56 & 55 \\
& L-S DQ & 76 & 29 & 34 \\
VAB-II-J & C-A DQ & 79 & 45 & 41 \\
& Adoptive behavior composite & 48 & 45 & 51 \\
& Communication & 63 & 34 & 58 \\
& Daily living skills & 47 & 54 & 61 \\
& Socialization & 38 & 36 & 45 \\
J-MCDls & Motor & 51 & 51 & 51 \\
& Words understood & 418 & 74 & 376 \\
& Words said & 413 & 3 & 180 \\
& Total gestures produced & 37 & 22 & 35 \\
\hline
\end{tabular}

PARS, pervasive developmental disorders autism society Japan rating scale; KSPD, Kyoto scales of psychological development 2001; DQ, developmental quotient; Full, total scale; $P-A$, physical-movement; L-S, language-sociability; C-A, cognitive-adaptive; VAB-II-J, Vineland adaptive behavior scales 2nd edition Japanese version; J-MCDIs, MacArthur Communicative Development Inventories, Japanese version.

\section{Positive Affect}

Taro and Sabu demonstrated almost the same levels of positive affect in both conditions. Jiro exhibited positive affect in 5\% of the intervals in the with automatic feedback condition and 35\% of the intervals in the without automatic feedback condition.

\section{Ball Contact}

All three children demonstrated increased levels of ball contact in the with automatic feedback condition. Specifically, the percentage of intervals with ball contact in the with automatic feedback condition was $65 \%$ for Taro, $95 \%$ for Jiro, and $60 \%$ for Sabu. In contrast, in the without automatic feedback condition, these figures decreased to 50,10 , and $45 \%$, respectively.

\section{Looking at the Therapist's Ball}

Similarly, all three children exhibited increased levels of looking at the therapist's ball in the with automatic feedback condition. Specifically, the percentage of intervals with looking at the therapist's ball during the with automatic feedback condition was $40 \%$ for Taro, $60 \%$ for Jiro, and $15 \%$ for Sabu. In contrast, during the without automatic feedback condition, these numbers decreased to 5,15 , and $0 \%$, respectively.

\section{EXPERIMENTAL STUDY}

\section{Participants}

All participants were recruited as volunteers through the Department of Psychology at Keio University. The participants were three boys with ASD, "Shiro," "Goro," and "Riku," between the ages of 3 and 6 years. Names of participants have been changed to protect the participants' identities. Informed consent was obtained from the parents before the children were included in the study. Table 2 displays the participants' characteristics.

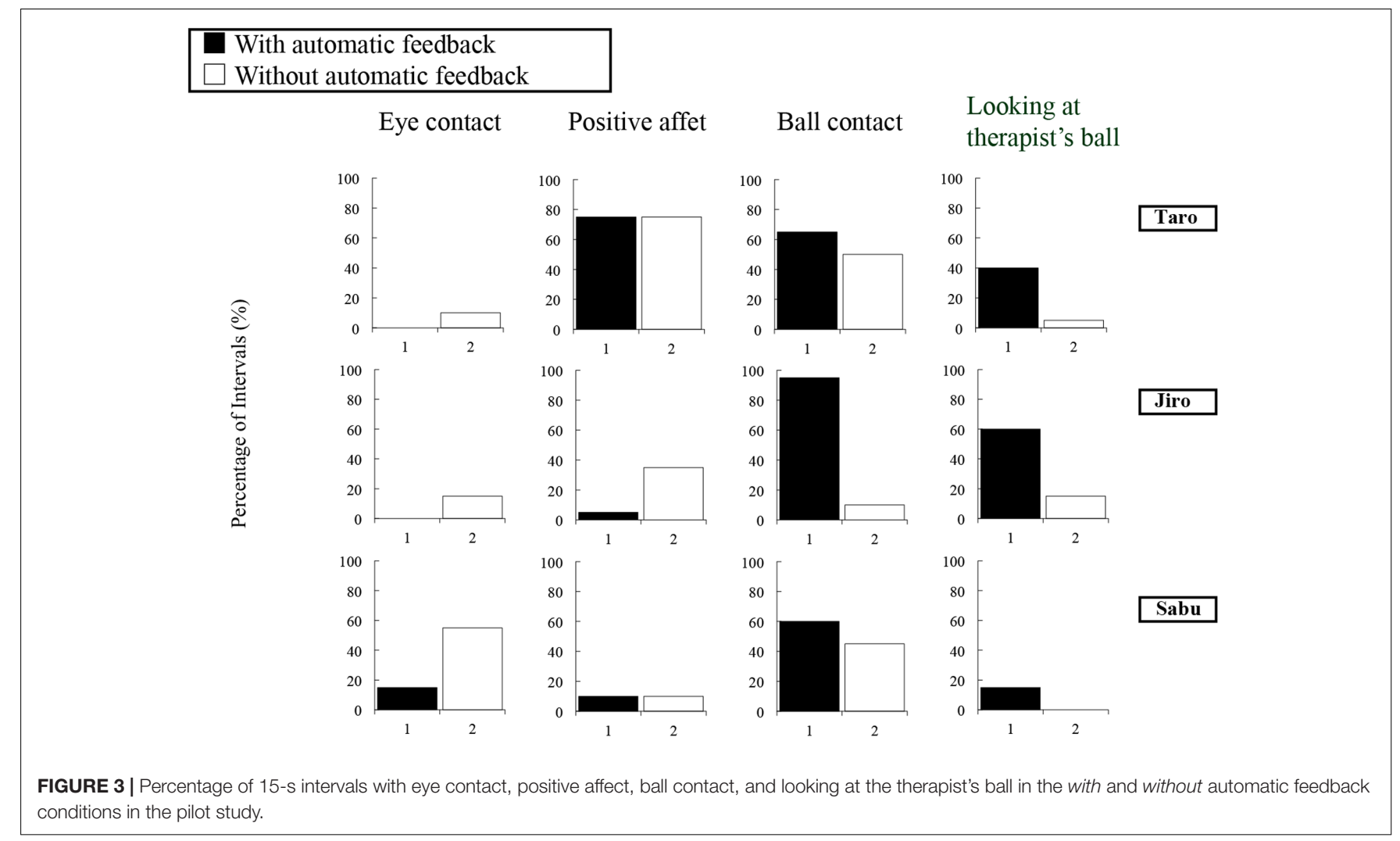


TABLE 2 | Participant profiles in the experiment.

\begin{tabular}{llccc}
\hline Child & & Shiro & Goro & Riku \\
\hline Chronological age & & $5 ; 8$ & $6 ; 8$ & $3 ; 8$ \\
PARS & Total peak symptom scale score & 52 & 46 & 28 \\
KSPD & Full DQ & 45 & 74 & 44 \\
& P-A DQ & 54 & 57 & 65 \\
& L-S DQ & 41 & 68 & 25 \\
VAB-II-J & C-A DQ & 49 & 79 & 45 \\
& Adoptive behavior composite & 55 & 53 & 49 \\
& Communication & 51 & 68 & 43 \\
& Daily living skills & 60 & 45 & 56 \\
& Socialization & 69 & 45 & 41 \\
J-MCDIs & Motor & 51 & 67 & 65 \\
& Words understood & 199 & 418 & 47 \\
& Words said & 181 & 338 & 11 \\
& Total gestures produced & 52 & 51 & 25 \\
\hline
\end{tabular}

PARS: pervasive developmental disorders autism society Japan rating scale; KSPD, Kyoto scales of psychological development 2001; DQ, developmental quotient; Full, total scale; $P-A$, physical-movement; L-S, language-sociability; C-A, cognitive-adaptive; VAB-II-J, Vineland adaptive behavior scales 2nd edition Japanese version; J-MCDIs, MacArthur Communicative Development Inventories, Japanese version.

\section{Design and Procedure}

The Council for Exceptional Children (CEC) Division of Research established a task force to develop guidelines for evidence-based practices (Odom et al., 2004). The task force identified four types of research methodologies: qualitative, correlational, experimental group, and single subject designs (Odom et al., 2004). Single subject designs have been used to compare the causal relationship between independent and dependent variables (Barlow et al., 2009). In this experiment, we used a single subject experimental design in a particular, rapidly changing reversal design (Cooper et al., 1990, 1993; Dunlap et al., 1991; Ishizuka and Yamamoto, 2016) over a total of two experimental days to compare the effects of lighting and vibration as automatic feedback. For all children, the experiment consisted of four 5-min sessions. Each participant had two 5-min sessions per day.

\section{Results}

For eye contact, the average observer agreement value was $80 \%$ (range 75-85\%); for positive affect, 88\% (range $85-90 \%$ ); for ball contact, 95\% (range 85-100\%); and for looking at the therapist's ball, $88 \%$ (range $75-100 \%$ ). Fidelity of implementation for socially/physically reinforcing the child's eye contact, positive affect, and approach to the therapist averaged $92 \%$; fidelity of implementation for socially/physically reinforcing the child's ball contact averaged 92\%; and fidelity of implementation for modeling and prompting ball play averaged $100 \%$. Results of the reversal analyses for each of the dependent variables are presented in Figure 4.

\section{Eye Contact}

Shiro exhibited eye contact with a mean of $20 \%$ of the intervals in the with automatic feedback condition and a mean of $12.5 \%$ in the without automatic feedback condition. Goro showed no eye contact in either condition. In the with automatic feedback condition, Riku exhibited eye contact for a mean of $40 \%$ of the intervals. On the other hand, in the without automatic feedback condition, his eye contact decreased to a mean of $20 \%$ across sessions.

\section{Positive Affect}

Shiro and Goro demonstrated a similar response pattern for positive affect. Specifically, in the initial with automatic feedback probe, they exhibited low positive affect. With the introduction of the without automatic feedback condition, their levels of positive affect increased to $45 \%$ (for Shiro) and $60 \%$ (for Goro) of the intervals. The reintroduction of the with automatic feedback condition was accompanied by a drop in positive affect levels to $5 \%$ and $10 \%$ of the intervals, respectively. The final without automatic feedback condition phase resulted in positive affect for 25 and $50 \%$ of the intervals, respectively, for the two boys.

In the first with automatic feedback probe, Riku exhibited positive affect in $15 \%$ of the intervals. Following the introduction of the without automatic feedback condition, his positive affect decreased slightly to $10 \%$ of the intervals. During the reintroduction of the with automatic feedback condition, Riku exhibited positive affect in $60 \%$ of the intervals. In the final without automatic feedback condition phase, Riku did not exhibit any positive affect.

\section{Ball Contact}

All three children demonstrated similar response patterns for ball contact. The initial with automatic feedback phase resulted in ball contact in 100\% (for Shiro), 85\% (for Goro), and 95\% (for Riku) of the intervals. With the introduction of the without automatic feedback condition, the levels of ball contact decreased to $75 \%, 40 \%$, and $5 \%$, respectively. The reintroduction of the with automatic feedback condition was accompanied by a rise in ball contact levels to 100,85 , and $95 \%$ of the intervals, respectively. The final without automatic feedback condition phase resulted in ball contact for 75,70 , and $30 \%$ of the intervals, respectively, for the three boys.

\section{Looking at the Therapist's Ball}

Shiro exhibited looking at the therapist's ball with a mean of $75 \%$ in the with automatic feedback condition and a mean of $47.5 \%$ in the without automatic feedback condition. For Goro, the means were $25 \%$ in the with automatic feedback condition and $10 \%$ in the without automatic feedback condition. In the with automatic feedback condition, Riku exhibited looking at the therapist's ball for a mean of $82.5 \%$ of the intervals. In contrast, during the without automatic feedback condition, his looking at the therapist's ball decreased to a mean of $15 \%$ across sessions.

\section{GENERAL DISCUSSION}

This study investigated the effects of automatic feedback in the form of colored lights and vibration produced via paired robotic devices, COLOLO, in social play and interaction in children with ASD. The frequency of ball contact and looking at 
With automatic feedback

Without automatic feedback

Eye contact

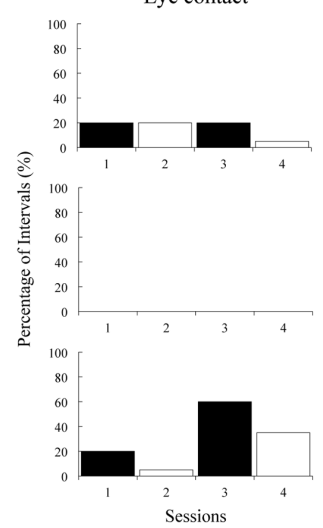

Positive affet
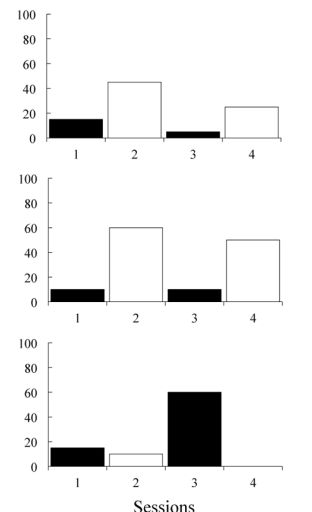
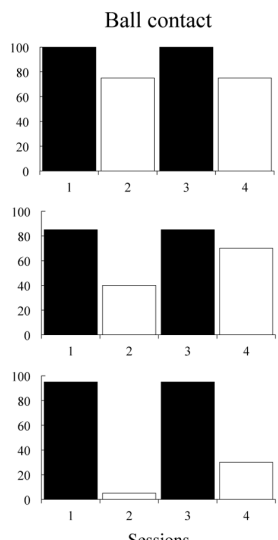

Looking at therapist's ball

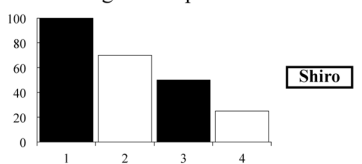

100
80
60
40

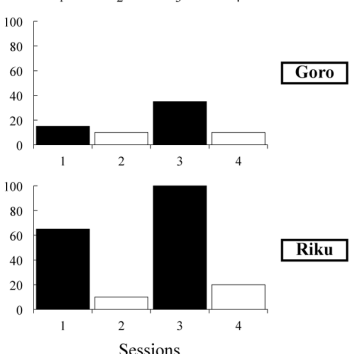

FIGURE 4 | Percentage of 15-s intervals with eye contact, positive affect, ball contact, and looking at the therapist's ball in the with and without automatic feedback conditions in the experimental study.

the therapist's ball were higher in the with automatic feedback condition than in the without automatic feedback condition, supporting Hypothesis 1. On the other hand, the frequencies of eye contact and positive affect for all children with ASD did not consistently increase or decrease in the with automatic feedback condition, thus the results indicated lack of support for both Hypothesis 2a and Hypothesis 2b. Therefore, when using the paired robotic devices, the children with ASD appear to have exhibited increases in social play behaviors using toys and but not increases in behaviors associated with social interaction.

Considering ball contact, Hypothesis 1 was positively supported. The findings are in lines with one of the pioneering works, which has demonstrated that a spherical mobile robot, Roball, may increase a child's interaction with a ball by providing automatic feedback consisting of motion, messages, sounds, and an illuminating interface (Michaud et al., 2005). This suggests that automatic feedback of vibration (tactile stimulus) might function as a reinforcer for ball contact behavior. However, we also used light feedback (visual stimulus). There is a possibility that light feedback also functions as a reinforcer for child's ball contact. Therefore, in a future study, we would evaluate which modality of feedback has a stronger effect on increasing ball contact.

Considering frequency of looking at the therapist's ball, the first Hypothesis was also positively supported. This indicates that attention to shared play materials might be increased by light feedback via paired robotic devices. Although we used vibration feedback, this feedback was not contingent upon the child looking at the therapist's ball, but contingent on the child looking at his own ball. Thus, light feedback provided via remotely connected paired devices may increase attention to the play materials of peers in children with ASD.

Concerning Hypotheses $2 \mathrm{a}$ and $2 \mathrm{~b}$ of the current study, our results did not support either of these hypotheses. Neither the child's eye contact nor their positive affect consistently increased as a result of the feedback in the form of light and vibration. The result can be easily interpreted because the feedback was not contingent upon the child's responses. In addition, however, increases in eye contact and positive affect were observed in the without automatic feedback condition for two children. A potential explanation for this outcome could be the frequency of the reinforcement provided by the therapist. As far as ecologically validity is concerned, in the procedure of this experiment, the therapist provided verbal/physical praise for the child's eye contact and positive affect throughout the session. This may have led to increased opportunity for praise for the therapist in the without automatic feedback condition in which the frequencies of child's ball contact was lower. To improve this aspect of the intervention, we recommended that future studies include the combined use of other wearable devices, such as an eye tracker (Ye et al., 2012) or a face reader device we have developed for detecting smiles from facial EMG signals (Gruebler and Suzuki, 2014), in order to provide contingent feedback for child eye contact and/or positive affect.

There were several limitations to the current study. First, we used a single subject experimental design with three children with ASD in this study, and this limits the generalizability of the results to the larger population due to limitations inherent in single subject experimental designs, such as absence of statistical analysis and inference. Further studies are required, including use of a group experimental design with larger sample sizes. Second, although we used an ABAB design to minimize carryover effects, because the experiment sessions were administered across 2 days, we were not able to eliminate ordering and novelty effects. It is possible that the novelty of the interaction affected the increase in the dependent variables on the 1st day (first set of $A B$ trials) and on the 2 nd day (second set of $\mathrm{AB}$ trials), due to the time that has elapsed between the first and the second session. Further studies must seek to eliminate ordering and novelty effects through blocked and longitudinal study designs. Third, we need to be cautious about interpreting the observed increases in children's ball contact and looking at the therapist's ball as the 
result of automatic feedback functioning as a reinforcer. It was unclear whether automatic feedback functioned as an antecedent stimulus or a reinforcer for children's ball contact and looking at the therapist's ball. Further research is warranted to identify the function of automatic feedback via the implementation of a yoked condition. Fourth, we only used the feedback of light (visual stimulus) and vibration (tactile stimulus). Future studies will be required to use other modalities, such as sound (auditory stimulus).

Nevertheless, the current findings establish that feedback via paired robotic devices can facilitate some aspects of social play behaviors in children with ASD, whereas previous studies have focused on examining differences between a human and a robot as an interaction partner (e.g., Costescu et al., 2015; Srinivasan et al., 2015; Simut et al., 2016), or investigating the effects of teaching by the robot (e.g., Billard et al., 2007; Warren et al., 2015). As Huskens et al. (2013) have suggested, it would be interesting to see more studies on this topic; in other words, there is a wide range of necessities for further investigation. While we are hopeful that clinical applications of paired robotic devices may demonstrate significant enhancement of social play for children with ASD at an early developmental stage, it is important to note that future research should reveal both whether and how the paired robotic devices contribute to increasing various forms of social play behaviors in children with ASD.

\section{REFERENCES}

American Psychiatric and Association (2000). Diagnostic and Statistical Manual of Mental Disorders. Washington, DC: American Psychiatric Association.

Barlow, D. H., Nock, M., and Hersen, M. (2009). Single Case Experimental Designs: Strategies for Studying Behavior for Change, 3rd Edn. Boston, MA: Pearson Education.

Baron-Cohen, S. (1987). Autism and symbolic play. Br. J. Dev. Psychol. 5, 139-148. doi: 10.1111/j.2044-835X.1987.tb01049.x

Barry, L. M., and Burlew, S. B. (2004). Using social stories to teach choice and play skills to children with autism. Focus Autism Other Dev. Disabil. 19, 45-51. doi: 10.1007/s10803-008-0628-9

Bass, J. D., and Mulick, J. A. (2007). Social play skill enhancement of children with autism using peers and siblings as therapists. Psychol. Sch. 44, 727-735. doi: $10.1002 /$ pits. 20261

Billard, A., Robins, B., Nadel, J., and Dautenhahn, K. (2007). Building robota, a mini-humanoid robot for the rehabilitation of children with autism. Assist. Technol. 19, 37-49. doi: 10.1080/10400435.2007.10131864

Boucher, J. (1999). Editorial: interventions with children with autism-methods based on play. Child Lang. Teach. Ther. 15, 1-5. doi: 10.1191/026565999 676029298

Charman, T., Baron-Cohen, S., Swettenham, J., Baired, G., Cox, A., and Drew, A. (2000). Testing joint attention, imitation, and play as infancy precursors to language and theory of mind. Cogn. Dev. 15, 481-498. doi: 10.1016/S08852014(01)00037-5

Cooper, L. J., Wacker, D. P., Millard, T., Derby, K. M., Cruikshank, B. M., and Rogers, L. (1993). Assessing environmental and medication variables in an outpatient setting: a proposed model and preliminary results with ADHD children. J. Dev. Phys. Disabil. 5, 71-85. doi: 10.1007/BF01046599

Cooper, L. J., Wacker, D. P., Sasso, G. M., Reimers, T. M., and Donn, L. K. (1990). Using parents as therapists to evaluate appropriate behavior of their children: application to a tertiary diagnostic clinic. J. Appl. Behav. Anal. 23, 285-296. doi: 10.1901/jaba.1990.23-285

\section{ETHICS STATEMENT}

This study was carried out in accordance with the recommendations of Keio University's Institutional Review Board with written informed consent from all parents of participants.

\section{AUTHOR CONTRIBUTIONS}

SM, EN, MH, JY, and KS designed the research. SM and EN performed the research. SM analyzed the data and wrote the article.

\section{FUNDING}

This work was supported by the CREST research project on Social Imaging, Japan Science and Technology Agency (JST) and a Grant-in-Aid for the Japan Society for the Promotion of Science (JSPS) Fellows.

\section{ACKNOWLEDGMENT}

The authors would like to thank Joseph P. McCleery for the English language review.

Costescu, C. A., Vanderborght, B., and David, D. O. (2015). Reversal learning task in children with autism spectrum disorder: a robot-based approach. J. Autism Dev. Disord. 45, 3715-3725. doi: 10.1007/s10803-014-2319-z

Diehl, J. J., Schmitt, L. M., Villano, M., and Crowell, C. R. (2012). The clinical use of robots for individuals with autism spectrum disorders: a critical review. Res. Autism Spectr. Disord. 6, 249-262. doi: 10.1016/j.rasd.2011.05.006

Dunlap, G., Kern-Dunlap, L., Clarke, S., and Robbins, F. R. (1991). Functional assessment, curricular revision, and severe behavior problems. J. Appl. Behav. Anal. 24, 387-397. doi: 10.1901/jaba.1991.24-387

Duquette, A., Michaud, F., and Mercier, H. (2008). Exploring the use of a mobile robot as an imitation agent with children with low-functioning autism. Auton. Robots 24, 147-157. doi: 10.1007/s10514-007-9056-5

Gruebler, A., and Suzuki, K. (2014). Design of a wearable device for reading positive expressions from facial EMG signals. IEEE Trans. Affect. Comput. 5, 227-237. doi: 10.1109/TAFFC.2014.2313557

Huskens, B., Palman, A., Van der Werff, M., Lourens, T., and Barakova, E. (2015). Improving collaborative play between children with autism spectrum disorders and their siblings: the effectiveness of a robot-mediated intervention based on Lego therapy. J. Autism Dev. Disord. 45, 3746-3755. doi: 10.1007/s10803-0142326-0

Huskens, B., Verschuur, R., Gillesen, J., Didden, R., and Barakova, E. (2013). Promoting question-asking in school-aged children with autism spectrum disorders: effectiveness of a robot intervention compared to a human-trainer intervention. Dev. Neurorehabil. 16, 345-356. doi: 10.3109/17518423.2012. 739212

Ikuzawa, M., Matsushita, Y., and Nakase, A. (eds). (2002). Kyoto Scale for Psychological Development 2001. Kyoto: Kyoto International Social Welfare Exchange Centre.

Ishizuka, Y., and Yamamoto, J. (2016). Contingent imitation increases verbal interaction in children with autism spectrum disorders. Autism 20, 1011-1020. doi: $10.1177 / 1362361315622856$

Ito, H., Tani, I., Yukihiro, R., Adachi, J., Hara, K., Ogasawara, M., et al. (2012a). Validation of an interview-based rating scale developed in Japan for 
pervasive developmental disorders. Res. Autism Spectr. Disord. 6, 1265-1272. doi: 10.1016/j.rasd.2012.04.002

Ito, H., Tani, I., Yukihiro, R., Uchiyama, K., Ogasawara, M., and Tsujii, M. (2012b). Development of the Japanese version of the vineland adaptive behavior scales. Second edition: reliability and validity of the maladaptive behavior scales. Seishin Igaku 54, 537-548.

Jahr, E., Eldevik, S., and Eikeseth, S. (2000). Teaching children with autism to initiate and sustain cooperative play. Res. Dev. Disabil. 21, 151-169. doi: 10.1016/S0891-4222(00)00031-7

Jarrold, C., Boucher, J., and Smith, P. (1993). Symbolic play in autism: a review. J. Autism Dev. Disord. 23, 281-307. doi: 10.1007/BF01046221

Jung, S., and Sainato, D. M. (2013). Teaching play skills to young children with autism. J. Intellect. Dev. Disabil. 38, 74-90. doi: 10.3109/13668250.2012.732220

Kamio, Y., Yukihiro, R., Adachi, J., Ichikawa, H., Inoue, M., Uchiyama, T., et al. (2006). Reliability and validity of the pervasive developmental disorder (PDD) autism society Japan rating scale: a behavior checklist for adolescents and adults with PDDs. Clin. Psychiatry 48, 495-505.

Le Couteur, A., Rutter, M., Lord, C., Rios, P., Robertson, S., Holdgrafer, M., et al. (1989). Autism diagnostic interview: a standardized investigator-based instrument. J. Autism Dev. Disord. 19, 363-387. doi: 10.1007/BF02212936

Lewis, V., and Boucher, J. (1988). Spontaneous, instructed and elicited play in relatively able autistic children. Br. J. Dev. Psychol. 6, 325-339. doi: 10.1111/j. 2044-835X.1988.tb01105.x

Lord, C., Rutter, M., and Le Couteur, A. (1994). Autism diagnostic interviewrevised: a revised version of a diagnostic interview for caregivers of individuals with possible pervasive developmental disorders. J. Autism Dev. Disord. 24, 659-685. doi: 10.1007/BF02172145

MacDonald, R., Clark, M., Garrigan, E., and Vangala, M. (2005). Using video modeling to teach pretend play to children with autism. Behav. Interv. 20, 225-238. doi: 10.1002/bin.197

Machalicek, W., Shogren, K., Lang, R., Rispoli, M., O’Reilly, M. F., Franco, J. H., et al. (2009). Increasing play and decreasing the challenging behavior of children with autism during recess with activity schedules and task correspondence training. Res. Autism Spectr. Disord. 3, 547-555. doi: 10.1016/j.rasd.2008.11.003

Michaud, F., Laplante, J. F., Larouche, H., Duquette, A., Caron, S., Letourneau, D., et al. (2005). Autonomous spherical mobile robot for child development studies. IEEE Trans. Syst. Man Cybern. A 35, 471-480. doi: 10.1109/TSMCA.2005. 850596

Morrison, R. S., Sainato, D. M., Benchaaban, D., and Endo, S. (2002). Increasing play skills of children with autism using activity schedules and correspondence training. J. Early Interv. 25, 58-72. doi: 10.1177/105381510202500106

Nunez, E., Matsuda, S., Hirokawa, M., Yamamoto, J., and Suzuki, K. (2016). “An approach to facilitate turn-taking behavior with paired devices for children with autism spectrum disorders," in Proceedings of the 25th IEEE International Symposium on Robot and Human Interactive Communication, Roman, 837-842. doi: 10.1109/roman.2016.7745216

Odom, S. L., Brantlinger, E., Gersten, R., Horner, R. D., Thompson, B., and Harris, K. (2004). Quality Indicators for Research in Special Education and
Guidelines for Evidence-Based Practices: Executive Summary. Arlington, VA: Council for Exceptional Children Division for Research.

Ogura, T. (2007). Early lexical development in Japanese children. Gengo Kenkyu 132, 29-53.

Robins, B., Dautenhahn, K., and Dickerson, P. (2009). "From isolation to communication: A case study evaluation of robot assisted play for children with autism with a minimally expressive humanoid robot," in Proceedings of the Second International Conferences on Advances in Computer-Human Interactions, ACHI'09 (Cancun: IEEE Computer Society Press), 205-211. doi: 10.1109/achi.2009.32

Simut, R. E., Vanderfaeillie, J., Peca, A., Van de Perre, G., and Vanderborght, B. (2016). Children with autism spectrum disorders make a fruit salad with Probo, the social robot: an interaction study. J. Autism Dev. Disord. 46, 113-126. doi: 10.1007/s10803-015-2556-9

Srinivasan, S. M., Park, I. K., Neelly, L. B., and Bhat, A. N. (2015). A comparison of the effects of rhythm and robotic interventions on repetitive behaviors and affective states of children with autism spectrum disorder (ASD). Res. Autism Spectr. Disord. 18, 51-63. doi: 10.1016/j.rasd.2015.07.004

Stahmer, A. C. (1995). Teaching symbolic play skills to children with autism using pivotal response training. J. Autism. Dev. Disord. 25, 123-141. doi: 10.1007/ BF02178500

Thorp, D. M., Stahmer, A. C., and Schreibman, L. (1995). Effects of sociodramatic play training on children with autism. J. Autism Dev. Disord. 25, 265-282. doi: 10.1007/BF02179288

Warren, Z. E., Zheng, Z., Swanson, A. R., Bekele, E., Zhang, L., Crittendon, J. A., et al. (2015). Can robotic interaction improve joint attention skills? J. Autism Dev. Disord. 45, 3726-3734. doi: 10.1007/s10803-013-1918-4

Williams, E., Reddy, V., and Costall, A. (2001). Taking a closer look at functional play in children with autism. J. Autism Dev. Disord. 31, 67-77. doi: 10.1023/A: 1005665714197

Wuff, S. B. (1985). The symbolic and object play of children with autism: a review. J. Autism Dev. Disord. 15, 139-148. doi: 10.1007/BF01531600

Ye, Z., Li, Y., Fathi, A., Han, Y., Rozga, A., Abowd, G. D., et al. (2012). “Detecting eye contact using wearable eye-tracking glasses," in Proceedings of the 2012 ACM Conference on Ubiquitous Computing, (New York, NY: ACM), 699-704. doi: $10.1145 / 2370216.2370368$

Conflict of Interest Statement: The authors declare that the research was conducted in the absence of any commercial or financial relationships that could be construed as a potential conflict of interest.

Copyright (c) 2017 Matsuda, Nunez, Hirokawa, Yamamoto and Suzuki. This is an open-access article distributed under the terms of the Creative Commons Attribution License (CC BY). The use, distribution or reproduction in other forums is permitted, provided the original author(s) or licensor are credited and that the original publication in this journal is cited, in accordance with accepted academic practice. No use, distribution or reproduction is permitted which does not comply with these terms. 